\title{
A signature of inflammatory proteins associated with ESRD in diabetes
}

\section{9 \\ inflammatory \\ processes \\ contributing \\ to the \\ development \\ of ESRD are \\ highly specific}

5)

is associated with the development of ESRD among three cohorts of patients with type 1 diabetes mellitus (T1DM) and type 2 diabetes mellitus (T2DM). "Our study points to the fact that inflammatory processes contributing to the development of ESRD are highly specific and that progression of the disease is not attributed to generalized inflammation as was often assumed," explains senior author Andrzej Krolewski. "Moreover, our study design, which comprised two cohorts of mostly white individuals and one cohort of Pima Indians, highlights that this process is universal across both T1DM and T2DM, early and late stages of the

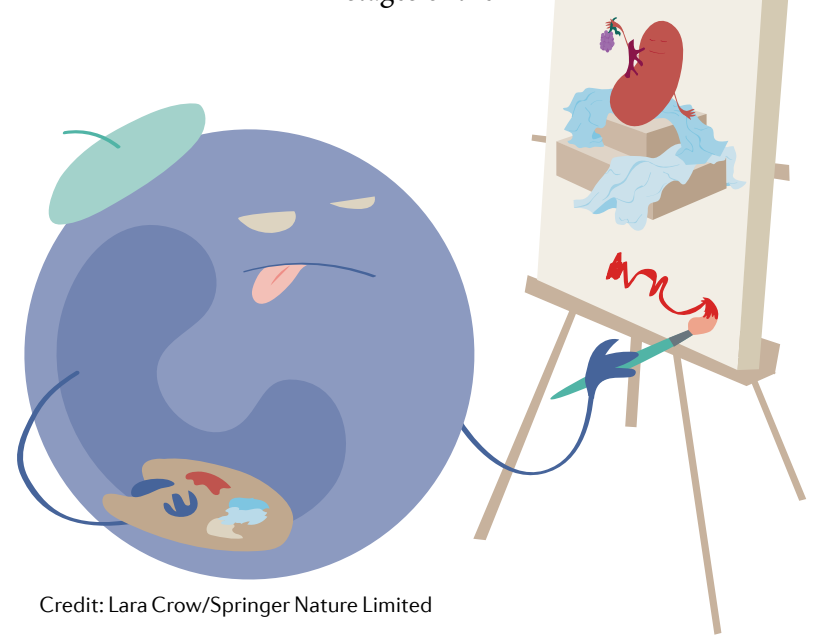

disease, and across differing ethnic backgrounds."

To identify plasma inflammatory proteins associated with the development of ESRD in patients with diabetes mellitus, first author Monika Niewczas and colleagues used a custom-designed aptamerbased proteomic platform to assess baseline plasma concentrations of 194 inflammatory proteins in patients with T1DM and T2DM from the Joslin kidney study ( $n=219$ and $n=144$, respectively), and in an independent cohort of 162 Pima Indians with T2DM. Participants from the Joslin cohorts had impaired kidney function at baseline, whereas those from the Pima Indian cohort had normal renal function at baseline; all participants included in the analyses developed ESRD within 10 years of study entry. These analyses led to the identification of 17 inflammatory proteins (termed the KRIS) that were associated with a 10 -year risk of developing ESRD. Of note, the protein signature was enriched in tumour necrosis factor receptor superfamily members. "We have previously identified important roles for TNFR1 and TNFR2 as robust predictors of progressive $\mathrm{DKD}$ - a finding that has been replicated by independent studies," says Niewczas. "Our current study reinforces the importance of these two proteins as they were among the proteins most strongly associated with ESRD in the signature. Furthermore, this study identified proteins that have never before been considered in the context of DKD."

In addition to their association with ESRD, the researchers identified a strong, inverse correlation between all 17 KRIS proteins and renal function decline in the two Joslin cohorts, whereas 14 of the KRIS proteins correlated with renal function decline in the Pima Indian cohort. Although the source of the inflammatory proteins was not identified, the researchers show that the proteins are produced systemically. "Our study provides convincing evidence that KRIS proteins do not originate from the kidney," notes Niewczas. "The majority of ongoing efforts in the field of DKD research have concentrated on the kidney as a major driver of disease processes. Our study sheds new light on the importance of systemic, previously underappreciated factors in disease pathogenesis."

Finally, the researchers show that although the angiotensin receptor blocker losartan does not affect levels of KRIS proteins, treatment with the JAK1/2 inhibitor baricitinib decreased levels of four KRIS proteins. "Greater understanding of the mechanisms that govern DKD will enable the identification of therapies that will ultimately lead to improved outcomes for patients with diabetes," says Krolewski. "A number of therapeutics that target pathways involving KRIS proteins are currently used for other indications; these agents could be investigated for their ability to prevent development of ESRD in patients with DKD.” In addition, the researchers plan to develop a focused, affordable assay to measure KRIS proteins in order to evaluate the potential prognostic value of this signature. "Our study provides strong evidence that more research is needed to evaluate the value of KRIS proteins as a biomarker of pharmacodynamic responses to different treatments," notes Krolewski.

Susan J. Allison

ORIGINAL ARTICLE Niewczas, M. A. et al. A signature of circulating inflammatory proteins and development of end-stage renal disease in diabetes. Nat. Med. https://doi.org/10.1038/ s41591-019-0415-5 (2019) 